\title{
SPY: A new scission point model based on microscopic ingredients to predict fission fragments properties
}

\author{
J.-F. Lemaître ${ }^{1,2}$, N. Dubray ${ }^{2}$, S. Hilaire ${ }^{2}$, S. Panebianco ${ }^{1}$, and J.-L. Sida ${ }^{1}$ \\ ${ }^{1}$ CEA Centre de Saclay, Irfu, 91191 Gif-sur-Yvette, France \\ ${ }^{2}$ CEA, DAM, DIF, 91297 Arpajon, France
}

\begin{abstract}
Our purpose is to determine fission fragments characteristics in a framework of a scission point model named SPY for Scission Point Yields. This approach can be considered as a theoretical laboratory to study fission mechanism since it gives access to the correlation between the fragments properties and their nuclear structure, such as shell correction, pairing, collective degrees of freedom, odd-even effects. Which ones are dominant in final state? What is the impact of compound nucleus structure? The SPY model consists in a statistical description of the fission process at the scission point where fragments are completely formed and well separated with fixed properties. The most important property of the model relies on the nuclear structure of the fragments which is derived from full quantum microscopic calculations. This approach allows computing the fission final state of extremely exotic nuclei which are inaccessible by most of the fission model available on the market.
\end{abstract}

\section{Introduction}

The fission process is too complex to be fully described within a single theory. There are many approaches to model this reaction but they can be crudely classified into two groups [1]. The first one is the phenomenological approach which is based on the physical interpretation of experimental data with quite simple theoretical tools which allows short computation time. However, this approach makes uses of many parameters fitted on data which limits the predictive power when going far from the region where data are available. The second one is the microscopic approach. It is based on a full quantum treatment of a microscopic nucleon-nucleon interaction to describe the system, both from the static and dynamic point of view. Therefore, even though the validity of this approach is ensured over the whole nuclear chart, the computation time can be a great limitation. Therefore, we chose an intermediate approach making use of one of the most up-to-date microscopic description of nuclear structure, implemented as an input in the framework of a rather simplified approach, a scission point model. This model, called SPY (Scission Point Yields) allows the determination of the fission fragment properties (yields, kinetic and excitation energies...) from a statistical treatment of all the static configurations which are energetically reachable at scission.

This is an Open Access article distributed under the terms of the Creative Commons Attribution License 2.0, which permits unrestricted use, distribution, and reproduction in any medium, provided the original work is properly cited. 


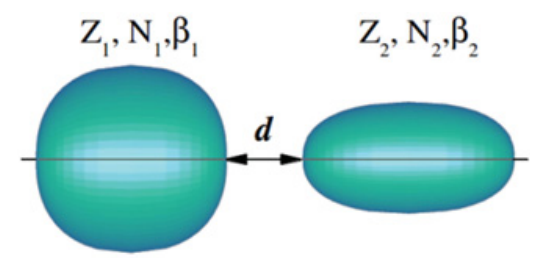

Figure 1. Schematic description of the two-fragment system at the scission point.

\section{The SPY model}

\subsection{General features}

In a scission-point model, very strong hypothesis are made concerning the fission process: the final state is determined from a static picture of the scission configuration. Indeed, the compound nucleus formation mechanism and the system dynamics from saddle to scission are not taken into account. In this approach, all fragments properties (mass, charge and deformation) are considered fixed at scission. As in the Wilkins model [2], the system at scission is modeled by two coaxial ellipsoids of revolution with sharp frontiers and separated by a fixed distance $d$ (Fig. 1). Each nucleus is characterized by its neutron $(\mathrm{N})$ and proton number $(\mathrm{Z})$ and its deformation parameter $(\beta)$. Thermodynamical equilibrium is supposed to be reached at scission, implying a statistical equilibrium among the system degrees of freedom. In addition the two fragments are considered to be isolated and all the states which are energetically reachable are equiprobable. Within these hypotheses, the scission configuration can be treated as a microcanonical statistical system in order to calculate average observables such as fission yields, mean kinetic and excitation energies of the fragments.

\subsection{Statistical treatment}

In a microcanonical statistical description, all energetically reachable states are equiprobable. Therefore, all system observables can be simply calculated by counting of available states for all the different fragmentations. Actually, the knowledge of all exact states is not necessary and only their number (or density) for a given configuration is needed. Since each configuration is fully defined by the intrinsic excitation of the two-fragment system, only two inputs are required: the available energy and the state density of each configuration. Moreover, we consider that the number of state accessible to the system is the product of the individual state densities of the two fragments.

For a given fissioning nucleus, the available energy of a scission configuration is calculated as a function of the deformation parameter of each fragment. This calculation is performed for all possible fragmentations, typically around one thousand.

The available energy calculation is based, as it is the case in Wilkins model, on an energy balance between the compound nucleus energy $\left(E_{C N}\right)$ and the intrinsic excitation energy of the system composed by the two nascent fragments (Eq. (1)). This latter contribution is obtained as the sum of the individual energy of the fragments and their interaction energy. The individual energy $\left(E_{H F B}\right)$ is extracted from the Amedee database [3, 4], which contains the potential energy as a function of the deformation for all the nuclei over the whole nuclear chart, calculated in the framework of Hartree Fock Bogoliubov (HFB) approach using the Gogny D1S nucleon-nucleon interaction [5]. This is one of the major improvements brought by SPY, compared to the existing scission-point models. It has the advantage to suppress all temperature parameters in the model and makes it more predictive. In order to keep a reasonable computing time, these microscopic data are integrated into SPY structure on the form of a large database. Finally, the interaction energy between the two fragments is composed of two terms: 

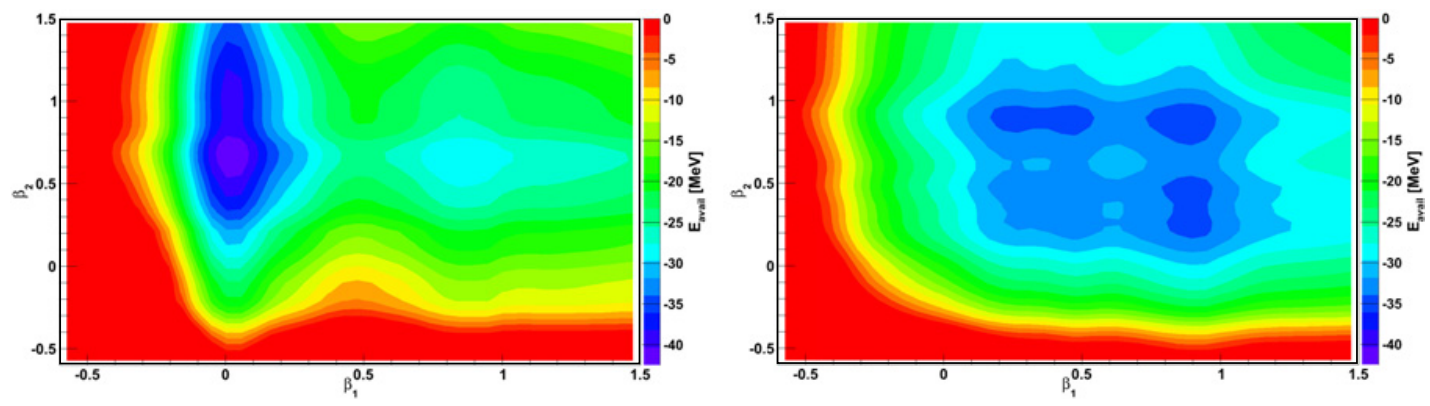

Figure 2. Available energy as a function of fragment deformations $\beta_{1}$ and $\beta_{2}$ for asymmetric fragmentation $\left({ }^{132} \mathrm{Sn}+{ }^{104} \mathrm{Mo}\right)$ on the left and for symmetric fragmentation $\left({ }^{118} \mathrm{Pd}+{ }^{118} \mathrm{Pd}\right)$ in ${ }^{235} \mathrm{U}$ thermal fission.

a Coulomb repulsion $\left(E_{C o u l}\right)$ [6] and a proximity nuclear interaction $\left(E_{\text {nucl }}\right)$ [7] (which is negligible with respect to the Coulomb repulsion). The interaction energy depends on the scission distance which, in all our calculation is kept fixed at $5 \mathrm{fm}$. A fragmentation is energetically achievable if available energy is negative.

$$
\begin{aligned}
E_{\text {avail }}= & E_{\mathrm{HFB} 1}\left(Z_{1}, N_{1}, \beta_{1}\right)+E_{\mathrm{HFB} 2}\left(Z_{2}, N_{2}, \beta_{2}\right)+E_{\mathrm{Coul}}\left(Z_{1}, N_{1}, \beta_{1}, Z_{2}, N_{2}, \beta_{2}, d\right) \\
& +E_{\text {nucl }}\left(Z_{1}, N_{1}, \beta_{1}, Z_{2}, N_{2}, \beta_{2}, d\right)-E_{\mathrm{CN}} .
\end{aligned}
$$

As an example, the available energy as a function of the two fragments deformation, calculated for two scission configurations in ${ }^{235} \mathrm{U}$ thermal fission, is presented in Figure 2. The most probable asymmetric fragmentation (Fig. 2 left) has its energy minimum at around $40 \mathrm{MeV}$ and it is characterized by one spherical fragment (the double magic ${ }^{132} \mathrm{Sn}$ ) and a rather deformed fragment $\left({ }^{104} \mathrm{Mo}\right)$. On the contrary, the symmetric splitting is characterized by a higher available energy (around $35 \mathrm{MeV}$ ) and two deformed fragments. The asymmetric configuration is energetically favored with respect to the symmetric one, as expected in ${ }^{235} \mathrm{U}$ thermal fission.

Once the available energy of the system is calculated, the computing of the reachable states is straightforward. Presently, the state density of the fragments used in SPY is a Fermi gas type from a generalized superfluid model [8]. This state density increases exponentially with the intrinsic energy $\varepsilon$ (Eq. (2)), where $a$ is the level density parameter and $A$ is the nucleus mass.

$$
\rho(\varepsilon) \propto e^{2 \sqrt{a \varepsilon}} \text { with } \mathrm{a} \approx \mathrm{A} / 8 .
$$

Since the system at scission is composed by two isolated subsystems which are the nascent fragments, the number of states $\pi$ for a given configuration is the product of the state densities of the two fragments $\left(\rho_{1}\right.$ and $\rho_{2}$ ) with $x$ the fraction of available energy in the fragment 1 and $(1-x)$ the fraction of available energy in the fragment 2 (Eq. (3)).

$$
\pi\left(Z_{1}, N_{1}, Z_{2}, N_{2}, \beta_{1}, \beta_{2}, x\right)=\rho_{1}\left(x\left|E_{\text {avail }}\right|\right) \rho_{2}\left((1-x)\left|E_{\text {avail }}\right|\right) \delta E^{2} .
$$

The probability of a given configuration is then given by the number of states accessible to this configuration, divided by the total number of states of the system; therefore it is proportional to $\pi$. Finally, the total probability $P$ to have a given fragmentation which is called the yield. It is obtained 


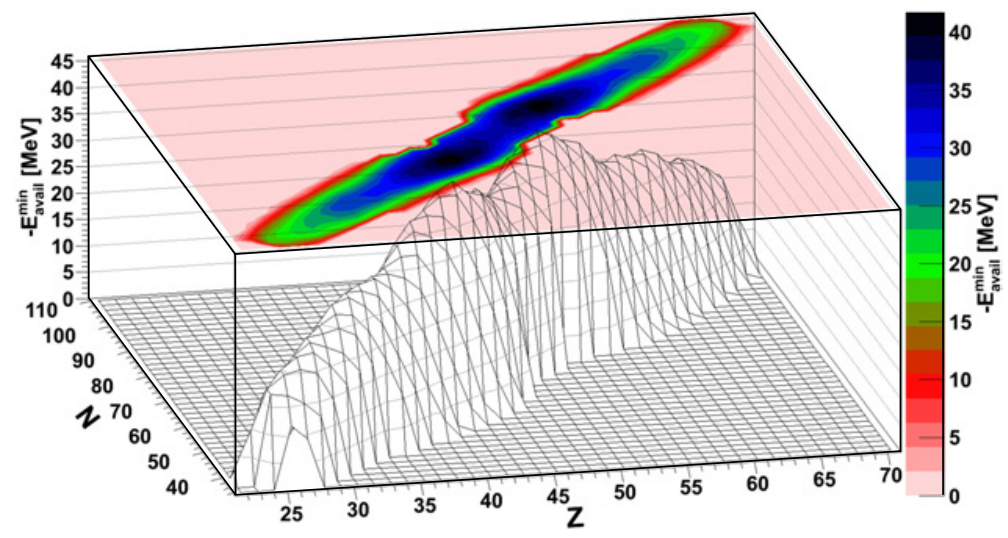

Figure 3. Minimal available energy as a function of the fragment proton $(Z)$ and neutron numbers $(N)$ for the thermal neutron induced fission of ${ }^{235} \mathrm{U}$.

by integrating over the two deformation parameters from quite large range deformation, from oblate to prolate deformation and all intrinsic energy repartitions (Eq. (4)).

$$
P\left(Z_{1}, N_{1}, Z_{2}, N_{2}\right)=\int_{-0.6}^{1.3} \int_{-0.6}^{1.3} \int_{0}^{1} \pi\left(Z_{1}, N_{1}, Z_{2}, N_{2}, \beta_{1}, \beta_{2}, x\right) d x d \beta_{1} d \beta_{2} .
$$

\section{Results}

As shown in the previous section, the energy balance contains already interesting information on the scission configurations because it gives access to the fragmentations which are energetically allowed. In addition, we note that, at the first order the more energy is available to the system, the more probable the fragmentation will be. Therefore, the minimum available energy is directly linked to the fragmentation yield. Based on this simple consideration, we could already explain the unexpected asymmetric fission of ${ }^{180} \mathrm{Hg}$ [9].

The same approach is here applied to the "standard" case of thermal neutron induced fission of ${ }^{235} \mathrm{U}$, where the maximum available energy (in absolute value) as a function of the fragment proton and neutron number is plotted in Figure 3. The distribution confirms that the most probable fission is asymmetric.

The statistical treatment described in the previous section is finally applied to obtain the fission yields (Fig. 4). We can notice that the two expected peaks appear around masses $\mathrm{A}=104$ and $\mathrm{A}=132$. This is clearly due to the fact that ${ }^{104} \mathrm{Mo}+{ }^{132} \mathrm{Sn}$ corresponds to the minimum available energy so it is the most probable fragmentation. Because statistical treatment with Fermi gas type state density does not introduce any structure on the available energy distribution. The comparison of the SPY results with evaluated fission yields is shown in Figure 4. We can see that SPY yields presents too sharp peaks, centered at 104 and 132, while in the data the peak are much broader and ranging, in the heavy peak from $\mathrm{A}=132$ and $\mathrm{A}=145$. This is due to two main effects. First, in our calculation the yields are mainly driven by the available energy at scission, which is strongly dominated by the fragment nuclear structure. This makes the double magic ${ }^{132} \mathrm{Sn}$ a too strong attractor since its high shell effect leads to the lowest available energy. Moreover, the lack of dynamics of the SPY model makes the peak too sharp since a static picture of the fission process prevents from exploring a rather large part of the phase space which is span by the system within its dynamics from saddle to scission. Finally, it should be noted that 


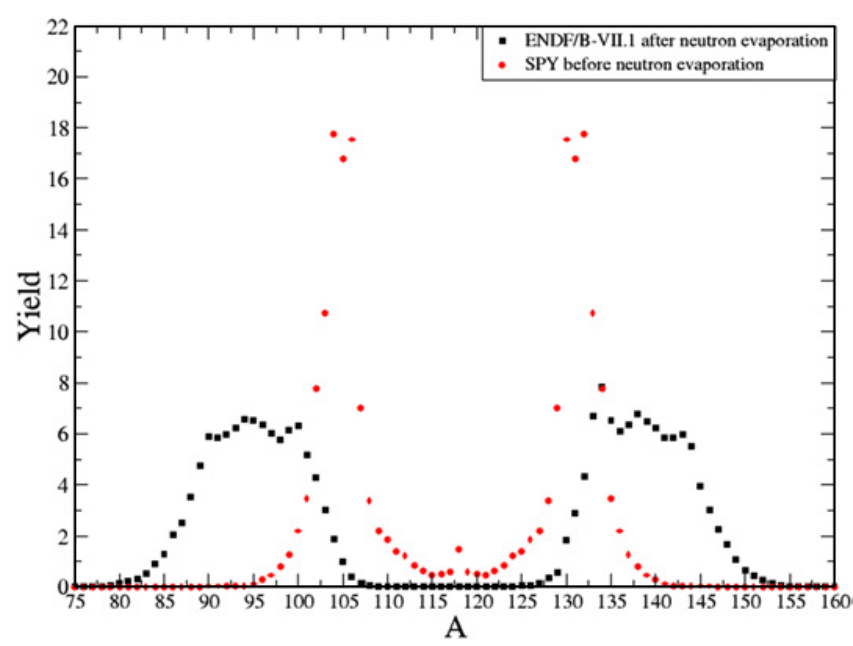

Figure 4. Comparison neutron thermal fission of ${ }^{235} \mathrm{U}$ yields between SPY model (red circle) and evaluated data (black square).

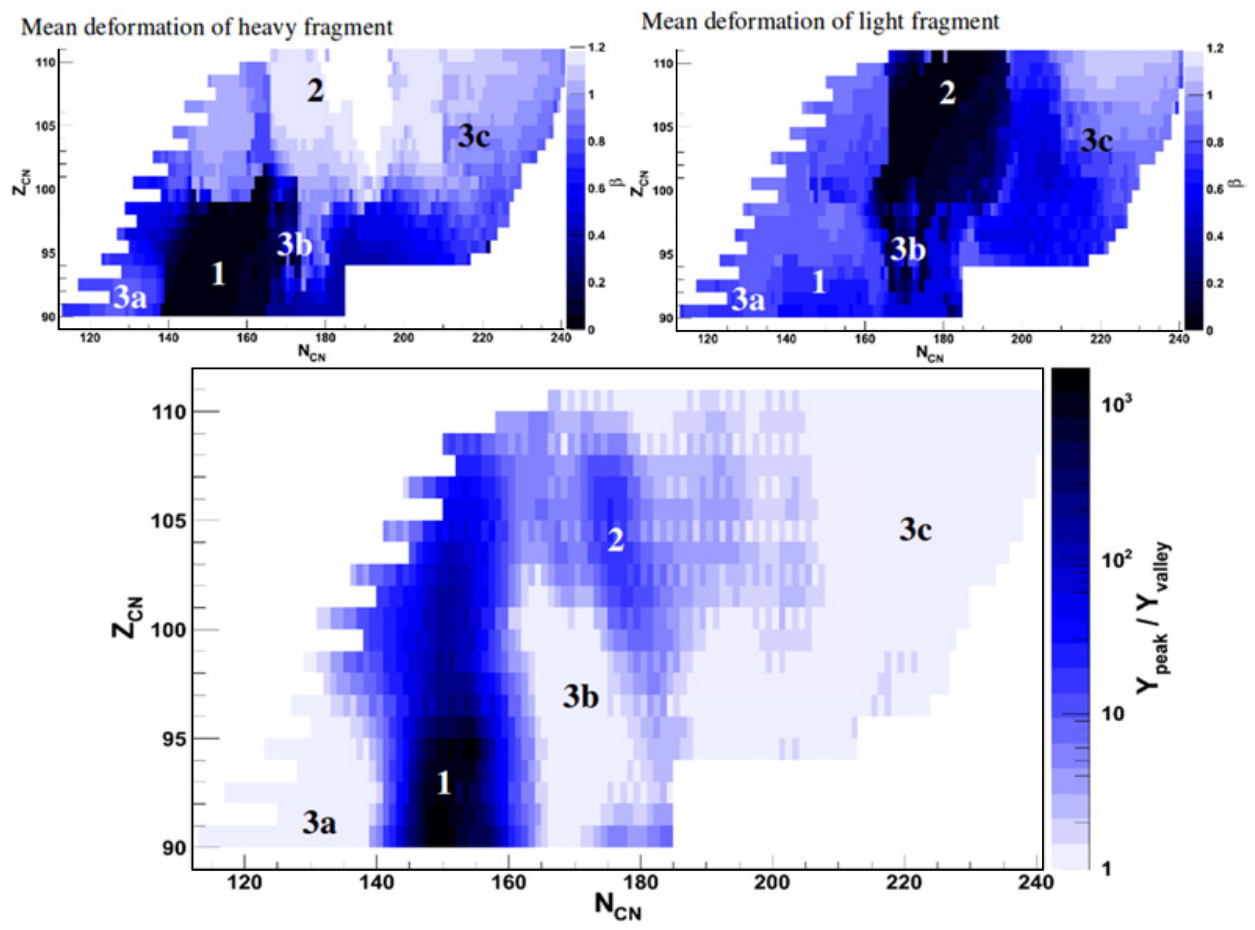

Figure 5. On the bottom, the peak over valley yields ratio from $Z_{C N}=90$ to $Z_{C N}=110$, from proton drip line to neutron drip line as a function of the neutron number and the proton number of the compound system $(\mathrm{CN})$. On the top, the deformation parameter $\beta$ of the most probable heavy (left) and light (right) fragment.

the evaluated data are the yields after neutron evaporation, while in SPY there is no evaporation. Despite the inability to accurately reproduce peaks positions, SPY model is able to determine the behavior of fragments in particular symmetric or asymmetric character of the fission. 
Indeed thanks to the use of microscopic data into a quite simple approach as a scission point model, the fragments properties of thousands of fissioning nuclei can be calculated in a rather fast time (few days with ten processors). The predictive power on the symmetric or asymmetric character of the fission brought by the microscopic description of the nuclei integrated in SPY gives access, for the first time, to the impact of the nuclear structure to the fission of nuclei that are inaccessible for the majority of the models available on the market. In particular, Figure 5 (bottom) shows the peak over valley yields ratio of about two thousand fissioning nuclei as a function of the compound nucleus proton and neutron numbers. The peak/valley yields ratio presents many structures which are correlated to the deformation of the fragments (Fig. 5 top).

First, there is the already discussed asymmetric fission region around $\mathrm{N}_{\mathrm{CN}}=150$ and $\mathrm{Z}_{\mathrm{CN}}<95$ : it is the actinide region (zone 1). The asymmetric fragmentation in this region is composed by a heavy fragment around the double magical ${ }^{132} \mathrm{Sn}$ which is spherical and a light slightly deformed light fragment. This tendency is in good agreement with experimental observations in this region of the nuclear chart. Then, there is another region of asymmetric fission around $\mathrm{Z}_{\mathrm{CN}}=105$ and $\mathrm{N}_{\mathrm{CN}}$ between 170 and 180 (zone 2) where now ${ }^{132} \mathrm{Sn}$ is the light fragment and the heavy fragment is even more deformed than the light fragment in the actinide region. Finally there are three regions of symmetric fission (zones 3a-3b-3c). The first two are related to very proton rich and very neutron rich actinides while the third one concerns very neutron rich exotic nuclei. In all these regions the two fragments are slightly deformed.

In conclusion the SPY model shows that the asymmetric and symmetric character of fission yields is mainly driven by nuclear structure of the fragments which plays the role of a stabilizer around magic nuclei. In addition the shell effects which are relevant for the fragmentation far from magicity, are not necessarily the ground state one but more often the deformed ones.

\section{Conclusions and perspectives}

SPY is a new scission-point model based on a static and statistical description of the fission process that makes use of one of the most up-to-date microscopic description of the nuclear structure. The interesting results obtained for the fission of actinides and light mercury isotopes makes us confident about this approach. Moreover, the richness of the microscopic input data opens the possibility to study the fission of unexploited regions of the nuclear chart. In particular, SPY predicts a new zone of asymmetric fission around $\mathrm{Z}_{\mathrm{CN}}=105$ and $\mathrm{N}_{\mathrm{CN}}$ between 170 and 180. In order to progress towards a more predictive model, there are some improvements which are presently under development. First, a large effort is being put on the integration of new state densities issued from the same microscopic calculations used in the available energy determination. This new input might partly counterbalance the too strong nuclear effects around magicity and will give to the model an overall coherence. In addition, the integration of the individual energy calculated with the Gogny D1M interaction is foreseen, together with the extension of the domain of validity of the model in terms of excitation energy (above $15 \mathrm{MeV}$ ). Finally, we are working in improving the definition of the scission point configuration in the model, thus to possibly wash out the impact of the scission distance in the calculation of the fission fragments characteristics.

\section{References}

[1] C. Wagnemans, The Nuclear Fission Process (CRC Press, 1991)

[2] B. D. Wilkins, E. P. Steinberg, and R. R. Chasman, Phys. Rev. C 14, 1832 (1976)

[3] S. Hilaire and M. Girod, Eur. Phys. J. A 33, 237 (2007)

[4] http://www-phynu.cea.fr/HFB-Gogny.htm 
[5] J. Decharge and D. Gogny, Phys. Rev. C 21, 1568 (1980)

[6] S. Cohen and W. Swiatecki, Ann. Phys. (NY) 19, 67 (1962)

[7] J. Błocki, J. Randrup, W. J. Swiatecki, and C. F. Tsang, Ann. Phys. (NY) 105, 427 (1977)

[8] A. V. Ignatyuk, J. L. Weil, S. Raman, and S. Kahane, Phys. Rev. C 47, 1504 (1993)

[9] S. Panebianco, J.-L. Sida, H. Goutte, J. F. Lemaître, N. Dubray and S. Hilaire, Phys. Rev. C 86, $064601(2012)$ 